\title{
Contribution of the Deviation from Perfect Gas Behavior to the Entropy and Heat Capacity. Water and Benzene
}

\author{
J. O. HALFORD \\ Chemistry Department, University of Michigan, Ann Arbor, Michigan
}

(Received October 4, 1948)

\begin{abstract}
The properties of water vapor are used to illustrate the point, previously found true for ethyl alcohol, that the modified Berthelot equation of state may not give a satisfactory evaluation of the deviation of thermodynamic properties from those of the hypothetical perfect gas. The entropy deviation for benzene, however, is accurate enough for most purposes, and the heat capacity is not too far out of line.

On limited information, it appears that the Berthelot equation may be satisfactory for the vapors of "normal" liquids up to limited pressures. It is recommended, however, that some attempt be made to check the properties by means of vapor densities, obtained directly or by the Clapeyron equation, before results based upon the Berthelot equation are accepted.
\end{abstract}

$\mathbf{I}^{\mathrm{T}}$ $T$ is of ten desired, for practical purposes, to calculate the entropy or heat capacity of a gaseous substance from spectroscopic data and molecular constants. Likewise, it may be desired to calculate these properties for the hypothetical perfect gas from thermal data and vapor pressures. In either case, it is necessary to evaluate the effect of the deviation from perfect gas behavior, an operation which, for maximum accuracy, would require extensive knowledge of the vapor density over a wide range of pressures and temperatures, or the equivalent in the form of an accurate and sufficiently detailed equation of state.

When adequate data are not available it has become broadly customary to use the modified Berthelot equation of state, with constants expressed in terms of the critical properties, to give an approximation to the entropy and heat capacity deviations.

The accuracy of this approximation was recently questioned by the author ${ }^{1}$ in a study of the entropy of ethyl alcohol, for which it developed that no reasonable combination of assigned molecular constants could be found which was consistent with the experimental entropy and the Berthelot equation over a sufficiently extended temperature range. Because the vapor pressures and heats of vaporization were apparently reliable and were inconsistent with volumes calculated from the Berthelot equation, it was most reasonable to assume that these volumes and the equation of state from which they were obtained were not acceptable.

In the ethyl alcohol case, the assignment of a potential barrier of at least $15000 \mathrm{cal} . / \mathrm{mole}$ to the hydroxyl group rotation was required if the Berthelot equation was used, but if the barrier evaluation was based upon the data at a low enough temperature, and larger deviations from perfect gas behavior at high temperature were recognized, a

\footnotetext{
1 J. O. Halford, J. Chem. Phys. 17, 111 (1949).
}

value near $3000 \mathrm{cal} . /$ mole was obtained. This illustrates how the inadequacy of the Berthelot equation can lead to serious errors in the description of hindered internal rotations.

The significance of the ethyl alcohol case to the general problem is obscure. It may mean only that inaccuracies will be found for the vapors of associated liquids, but it could be true that serious errors would appear elsewhere as well.

The purpose of the present paper is to compare the correct entropy and heat capacity deviations with those obtained from the Berthelot equation for two substances, water and benzene, chosen as examples, respectively, of the associated and the normal liquid. There are, in fact, very few compounds for which there are enough data to support an accurate calculation of the deviations. In the case of ethane, heat capacity increments calculated by Kistiakowsky and Rice ${ }^{2}$ from the vapor densities of Eucken and Parts ${ }^{3}$ are substantially the same as those obtained from the Berthelot equation.

\section{WATER}

The required information for water vapor appears in a recent article by Keyes, ${ }^{4}$ who tabulates the second, third, and fourth virial coefficients and their derivatives as functions of the temperature and gives the explicit expression for the entropy correction. The units at the head of the columns of virial coefficients, however, cannot be correct, the expressions for the coefficients in the entropy equation are not clearly bracketed, and the true entropy coefficients appear to be the negatives of the indicated ones. In using the Keyes expression, therefore, it has been assumed that the virial coefficients are correct for energy in $\mathrm{cc}$ atmos., the missing brackets

${ }^{2}$ G. B. Kistiakowsky and W. W. Rice, J. Chem. Phys. 5, 281 (1939).

3 A. Eucken and A. Parts, Zeits. f. physik. Chemie 20B, 189 (1933).

${ }_{4}^{4}$ F. G. Keyes, J. Chem. Phys. 15, 602 (1947). 
TABLE I. Entropy of gas imperfection for water vapor at saturation pressures (cal./mole deg.).

\begin{tabular}{lllc}
\hline$T\left({ }^{\circ} \mathrm{K}\right)$ & $p$ (at.) & $S^{*}-S$ & $\begin{array}{c}S^{*}-S \\
\text { (Berthelot) }\end{array}$ \\
\hline 273.2 & 0.006026 & 0.006 & 0.001 \\
283.2 & 0.01211 & 0.009 & 0.001 \\
293.2 & 0.02306 & 0.013 & 0.002 \\
303.2 & 0.04187 & 0.018 & 0.003 \\
313.2 & 0.07281 & 0.025 & 0.005 \\
323.2 & 0.12179 & 0.035 & 0.008 \\
333.2 & 0.19667 & 0.045 & 0.011 \\
343.2 & 0.30764 & 0.060 & 0.016 \\
353.2 & 0.46752 & 0.077 & 0.022 \\
363.2 & 0.69201 & 0.098 & 0.030 \\
373.2 & 1.0000 & 0.122 & 0.040 \\
383.2 & 1.4137 & 0.150 & 0.052 \\
393.2 & 1.9589 & 0.183 & 0.067 \\
403.2 & 2.6650 & 0.222 & 0.085 \\
413.2 & 3.5648 & 0.268 & 0.105 \\
423.2 & 4.6952 & 0.313 & 0.129 \\
433.2 & 6.100 & 0.352 & 0.157 \\
443.2 & 7.817 & 0.410 & 0.187 \\
453.2 & 9.895 & 0.478 & 0.220 \\
\hline \hline
\end{tabular}

have been supplied to the expressions for the coefficients, and the sign of the entire entropy effect has been reversed. The increments finally obtained have been added to the perfect gas entropy and found to agree with the entropy values of Osborne, Stimson, and Ginnings ${ }^{5}$ within precisely the same limits as stated in the last paragraph of the paper by Keyes. Units and conversion factors correspond to the following values of the gas constant: $1.9872 \mathrm{cal} . / \mathrm{mole}$ deg., 82.057 cc atmos./mole deg., 8.3130 int. joule/mole deg. In checking against the entropy values of Osborne, Stimson, and Ginnings, the results obtained from the Keyes' equation were converted to calories defined by the conversion factor $0.238891 \mathrm{cal} . /$ int. joule.

Table I shows the entropy of gas imperfection for water vapor at the saturation pressure from $273.2^{\circ} \mathrm{K}$ to $453.2^{\circ} \mathrm{K}$. The second column gives the vapor pressure, the third the entropy correction from the Keyes data and the fourth the corresponding increment from the modified Berthelot equation.

TABLE II. Estimated heat capacity of gas imperfection for water vapor at one atmosphere (cal./mole deg.).

\begin{tabular}{ccc}
\hline \hline$T\left({ }^{\circ} \mathrm{K}\right)$ & $C-C^{*}$ & $\begin{array}{c}C-C^{*} \\
\text { (Berthelot) }\end{array}$ \\
\hline 303.2 & 16.19 & 0.22 \\
313.2 & 8.42 & 0.21 \\
323.2 & 5.20 & 0.20 \\
333.2 & 3.18 & 0.17 \\
343.2 & 1.93 & 0.16 \\
353.2 & 1.52 & 0.14 \\
363.2 & 1.12 & 0.13 \\
373.2 & 0.86 & 0.12 \\
383.2 & 0.67 & 0.10 \\
393.2 & 0.54 & 0.09 \\
403.2 & 0.44 & 0.08 \\
\hline \hline
\end{tabular}

${ }^{5}$ N. S. Osborne, H. F. Stimson, and D. C. Ginnings, J. Res. Nat. Bur. Stand. 23, 261 (1939).
The vapor pressures up to $423.2^{\circ}$ are taken from Keyes, those at higher temperatures come from the International Critical Tables. The last three entropy increments have been obtained by difference from the entropies of Osborne, Stimson, and Ginnings and the Keyes' expression for the perfect gas, and may be low by one or two percent. The critical constants used for the last column are $T_{c}=643.2^{\circ} \mathrm{K}$, $p_{c}=217.7$ atmos.

Whether or not the Berthelot values are acceptable depends upon the achievable accuracy of the result. In relation to the limits $\pm 0.05 \mathrm{cal} . /$ mole deg. placed by Giauque and Stout ${ }^{6}$ upon their value for the liquid at $298.2^{\circ} \mathrm{K}$, the error introduced by the Berthelot equation is negligible (less than 10 percent of the total) only at the lowest temperature where the increment itself is practically negligible. The error becomes equal to the total estimated error in the region of $345^{\circ} \mathrm{K}$ where the pressure is about 0.35 atmos.

A rough picture of the situation in connection with the heat capacity can also be obtained from the data. It would appear to be very difficult to achieve accuracy in the heat capacity of gas imperfection. For example, if the simplified virial equation of state,

$$
p V=R T+B p
$$

is considered, the heat capacity increment is given by $\delta C_{p} / \delta p=-T \delta^{2} B / \delta T^{2}$. The coefficient $B$, the difference between the perfect gas volume and the actual volume, is difficult to obtain with accuracy, and, consequently, the second derivative with the temperature will be subject to large errors.

For the present purpose, the entropy increment at one atmosphere has been calculated by the procedure used to set up Table I, and the heat capacity increment has been obtained as a rough graphical estimate of the equivalent quantity $T(\delta S / \delta T)_{p}$. Table II shows the result.

Obviously the Berthelot equation is not satisfactory. While it prescribes a fixed value of three for the heat capacity/entropy ratio, this ratio is variable and much larger. For example, the heat capacity increment at the boiling point is seven times as large as the entropy increment.

\section{BENZENE}

Scott, Waddington, Smith, and Huffman ${ }^{7}$ have evaluated the coefficient $B$ in Eq. (1) as

$$
B(c c)=-202-53.5 \exp [950 / T] .
$$

This equation has been made consistent with their measured heat capacities and those calculated from

\footnotetext{
${ }^{6}$ W. F. Giauque and J. W. Stout, J. Am. Chem. Soc. 58, 1144 (1936)

${ }^{7}$ D. W. Scott, G. Waddington, J. C. Smith, and H. M. Huffman, J. Chem. Phys. 15, 565 (1947).
} 
molecular constants, subject to the validity of their interpretation of the effect of anharmonicity upon the vibrational entropy. The volumes are also in fair agreement with values obtained from heats of vaporization and vapor pressures by means of the Clapeyron equation.

If Eq. (2) is accepted as correct, then the entropy and heat capacity increments are independent of the pressure and are given by

$$
S^{*}-S=p d B / d T
$$

and

$$
C-C^{*}=-p T d^{2} B / d T^{2}=(2+950 / T)\left(S^{*}-S\right) \text {. }
$$

The entropy and heat capacity increments have been evaluated along the vapor pressure curve, using Young's vapor pressures, as quoted by Fiock, Ginnings, and Holton, ${ }^{8}$ as accurate enough for present purposes. The results are presented in Table III. The critical constants used to obtain the Berthelot values are $T_{c}=561.7^{\circ} \mathrm{K}$ and $p_{c}=47.7$ atmos.

Here the Berthelot equation gives a remarkably good evaluation of the entropy effect throughout the entire range and is in exact agreement just above the boiling point. The heat capacity, however, is not very good at the lower temperatures but is approaching agreement as the temperature increases.

\section{CONCLUSIONS}

From the foregoing examples, including the cases of ethane and ethyl alcohol to which reference has been made, it would appear that the entropy of gas imperfection can be obtained with fair accuracy for the vapors of normal liquids from the modified Berthelot equation of state, but that for water and the lower alcohols, an evaluation by this equation may not be satisfactory.

Heat capacity increments for the associated compounds, when obtained from the Berthelot equation, are seriously in error, and they are not highly dependable even in the case of benzene.

Under the circumstances, it would appear advisable to limit the application of the Berthelot equation to the entropy increment for non-associated substances to pressures well below one atmosphere and to use this equation, or any other selected in a similarly arbitrary manner, for associated substances only when it clearly indicates that the increment is negligible relative to the limits of error from other sources.

Because the number of accurately demonstrable cases is not sufficient to support a broad generalization, it would be most conservative, even for the vapors of normal liquids, to use the Berthelot

${ }^{8}$ E. F. Fiock, D. C. Ginnings, and W. B. Holton, J. Res. Nat. Bur. of Stand. 6, 881 (1931).
TABLE III. Entropy and heat capacity of gas imperfection for benzene vapor at saturation pressures (cal./mole deg.).

\begin{tabular}{lllclc}
\hline \hline & & & & \\
\hline & & & & \\
\hline & & & \\
$S^{*}-S$ & & & $C-C^{*}$ \\
273.2 & 0.0349 & 0.019 & 0.011 & 0.102 & 0.032 \\
283.2 & 0.0598 & 0.026 & 0.016 & 0.141 & 0.049 \\
293.2 & 0.0982 & 0.036 & 0.024 & 0.188 & 0.074 \\
303.2 & 0.1556 & 0.048 & 0.035 & 0.245 & 0.104 \\
313.2 & 0.2383 & 0.062 & 0.048 & 0.312 & 0.145 \\
323.2 & 0.3539 & 0.079 & 0.065 & 0.389 & 0.196 \\
333.2 & 0.5113 & 0.098 & 0.086 & 0.476 & 0.258 \\
343.2 & 0.7203 & 0.120 & 0.111 & 0.571 & 0.333 \\
353.2 & 0.9916 & 0.146 & 0.140 & 0.682 & 0.420 \\
363.2 & 1.337 & 0.171 & 0.174 & 0.787 & 0.521 \\
373.2 & 1.769 & 0.199 & 0.212 & 0.906 & 0.635 \\
383.2 & 2.300 & 0.230 & 0.256 & 1.030 & 0.764 \\
393.2 & 2.945 & 0.263 & 0.303 & 1.160 & 0.907 \\
403.2 & 3.717 & 0.300 & 0.353 & 1.294 & 1.059 \\
& & & & & \\
\hline \hline
\end{tabular}

equation only when some supporting data are available.

\section{USE OF THE CLAPEYRON EQUATION}

The virial coefficient of Eq. (1) is given by the exact Clapeyron equation as

$$
B=V(l)+\Delta H /(T d p / d T)-R T / p
$$

in which $V(l)$ is the molal volume of the liquid at saturation and is negligible at low pressures. When the vapor pressure and heat of vaporization are known with enough accuracy as functions of the temperature, the deviation volume $B$ can be obtained in relation to the temperature. If it is assumed, as in the case of benzene, that $B$ is a function only of the temperature, the entropy and heat capacity effects can be obtained by Eqs. (3). Since, however, both $\Delta H$ and $d p / d T$ are hard to measure accurately, and the error in their ratio will be magnified by the subtraction of the perfect gas volume, it will be difficult to obtain $B$ accurately, and progressively more difficult to obtain the first and second temperature derivatives.

A number of trials with data of ordinary accuracy to be found in the literature have indicated that the highest accuracy will probably be obtained near the boiling point and that at low temperatures and pressures, the results are not dependable. It is therefore suggested that for most purposes it will be satisfactory to evaluate the entropy and heat capacity effects in the region of the boiling point, and to project them into the region of lower temperatures along some arbitrary curve which brings them near zero at temperatures of the order of $100^{\circ}$ below the boiling point.

A variation of the method of using the Clapeyron equation is possible which may at some time prove to be useful. Lewis and Randall ${ }^{9}$ have shown that, for a gas obeying Eq. (1), if $B$ is a function of the

${ }^{9}$ G. N. Lewis and M. Randall, Thermodynamics and the Free Energy of Chemical Substances (McGraw-Hill Book Company, New York, 1923), p. 197. 
temperature alone and is small relative to the volume, the fugacity, defined by the relation $F=R T \ln f$, can be expressed as

$$
f=p^{2} / p(\text { ideal })=p V / V(\text { ideal }) \text {. }
$$

The fugacity can be calculated from vapor densities or from the volumes obtained by means of the Clapeyron equation and, since this property must represent the pressure at which the hypothetical perfect gas would be at equilibrium with the liquid, it follows, if the liquid volume is negligible, that

$$
\Delta S^{*}=R T d \ln f / d T .
$$

Equation (6) gives the entropy of vaporization to the ideal gas at the pressure $f$, and its use should be the practical equivalent of the usual separate evaluation of the entropies of vaporization and of gas imperfection.

\title{
Thermal Diffusion in Hydrogen-Hydrocarbon Mixtures
}

\author{
H. G. Drickamer, S. L. Downey, and N. C. Pierce, \\ Department of Chemisiry, University of Illinois, Urbana, Illinois
}

(Received October 8, 1948)

\begin{abstract}
Thermal diffusion data have been obtained on a series of mixtures of hydrogen plus hydrocarbons. The results indicate that "collision diameter" for these unlike molecules is considerably smaller than the mean collision diameter of the pure compounds. This is consistent with the high critical pressure of such mixtures.
\end{abstract}

\section{NOMENCLATURE}

$\alpha$-Thermal diffusion ratio

$D_{12}$ - Coefficient of ordinary diffusion

$\epsilon-$ Difference between energy of separated molecules and configuration for which they have maximum attraction

$E$-Potential energy

$m$-Molecular mass

$M_{1}-\left(m_{1} / m_{1}+m_{2}\right)$

$r$-Intermolecular distance

$r_{0}$ - "Collision diameter"-distance of separation from zero interaction energy

$T_{1}, T_{2}-$ Temperatures of upper and lower bulb respectively

$T_{m}$-Average temperature

$v_{1}, v_{2}-$ Convection velocities of species 1,2

$W^{l}$ r-Integral involving collision cross sections

$x_{1}, x_{2}-$ Mole fractions of species 1,2

\section{THEORY}

$T$

HE thermal diffusion ratio $\alpha$ is defined by the equation,

$$
x_{1} x_{2}\left(v_{1}-v_{2}\right)=D_{12}\left(-\operatorname{grad} x_{1}+x_{1} x_{2} \alpha \operatorname{grad} \ln T\right) \text {. }
$$

From the kinetic theory of gases Chapman and Cowlingl give the following relationship for $\alpha$ :

$$
\alpha=5(c-1) \frac{x_{1} S_{1}+x_{2} S_{2}}{x_{1} Q_{1}+x_{2} Q_{2}+x_{1} x_{2} Q_{12}}
$$

which for 50-50 mixtures of gases reduces to

$$
\begin{aligned}
\alpha & =10(c-1) \frac{S_{1}+S_{2}}{Q_{1}+Q_{2}+Q_{12}} \\
& =10(c-1) G,
\end{aligned}
$$

IS. Chapman and T. G. Cowling, Mathematical Theory of Non-uniform Gases (Cambridge University Press, Teddington, 1939). where

$$
\begin{aligned}
& S_{1}=\frac{M_{1}}{M_{2}}\left(\frac{r_{0}}{r 0_{12}}\right)^{2} \frac{W_{1}^{(2)}(2)^{\sqrt{2} A}}{W_{12}^{(2)}(2)} \\
& -M_{2}\left\{3\left(M_{2}-M_{1}\right)+4 M_{1} A\right\}, \\
& Q_{1}=\frac{\sqrt{2} A}{M_{2}^{1}}\left(\frac{r 0_{1}}{r 0_{12}}\right)^{2} \frac{W_{1}^{(2)}(2)}{W_{12}^{(2)}(2)} \\
& \times\left\{6 M_{2}^{2}+5 M_{1}^{2}-4 M_{1}^{2} B+8 M_{1} M_{2} A\right\}, \\
& Q_{12}=\left(M_{1}-M_{2}\right)^{2}(5-4 B)+4 M_{1} M_{2} A(11-4 B) \\
& +4\left(\frac{r 0_{1}}{r 0_{12}}\right)^{2}\left(\frac{r_{02}}{r 0_{12}}\right)^{2} \\
& \times \frac{A^{2}}{\left(M_{1} M_{2}\right)^{\frac{1}{2}}} \frac{W_{1}^{(2)}(2) W_{2}^{(2)}(2)}{\left(W_{12}^{(2)}(2)\right)^{2}}, \\
& A=\left(W_{12}^{(2)}(2) / 5 W_{12}^{(1)}(1)\right) \text {, } \\
& B=\left(5 W_{12}^{(1)}(2)-W_{12}^{(1)}(3) / 5 W_{12}^{(1)}(1)\right) \text {, } \\
& C=\left(2 W_{12}^{(2)}(2) / 5 W_{12}^{(1)}(1)\right) \text {. }
\end{aligned}
$$

$S_{2}, Q_{2}$ can be obtained by reversing subscripts.

The nomenclature has been modified slightly to correspond to that used by Hirschfelder, Bird, and Spotz. ${ }^{2}$

The evaluation of the integrals $W^{(l)}(r)$ involves the use of the collision cross section and therefore depends on the intermolecular force law assumed. Hirschfelder et al., ${ }^{2}$ have evaluated these integrals

2 J. O. Hirschfelder, R. B. Bird, and E. Spotz, J. Chem. Phys. 16, 968 (1948). 